\title{
Umbilical Cord Venous Lactate for Predicting Arterial Lactic Acidemia and Neonatal Morbidity at Term
}

\author{
Methodius G. Tuuli, MD, MPH${ }^{1}$, Molly J. Stout, MD, MSCI ${ }^{1}$, George A. Macones, MD, MSCE${ }^{1}$, \\ and Alison G. Cahill, MD, MSCl ${ }^{1}$ \\ ${ }^{1}$ Department of Obstetrics and Gynecology Washington University School of Medicine
}

\begin{abstract}
Objective-To estimate the utility of umbilical venous lactate, more readily available than umbilcal cord arterial lactate, for predicting arterial lactic acidemia and neonatal outcomes at term.

Methods-This was a prospective cohort study of consecutive, non-anomalous, singleton, term births following labor in a large academic medical center (2009 - 2014). Umbilical arterial and venous lactate were measured immediately after delivery, prior to knowledge of neonatal outcomes. The outcome measures were arterial lactic acidemia $(>3.9 \mathrm{mmol} / \mathrm{L})$ and a composite neonatal outcome consisting of neonatal death and any of a number of neonatal morbidities including intubation, mechanical ventilation, meconium aspiration syndrome, hypoxic-ischemic encephalopathy, and therapeutic hypothermia. Predictive ability of venous lactate was estimated using the area under the receiver-operating characteristics curve (AUC).
\end{abstract}

Results-Among 7,741 births, venous lactate was strongly predictive of arterial lactic acidemia (AUC $=0.958$ ). The 'optimal' cut-point of venous lactate for predicting both arterial lactic acidemia and the composite neonatal outcome was $3.4 \mathrm{mmol} / \mathrm{L}$ This predicted arterial lactic acidemia with sensitivity of $87.0 \%$ and specificity of $91.3 \%$. Positive and negative predictive values were $79.9 \%$ and $94.7 \%$, respectively. The composite neonatal outcome occurred in 104 neonates $(1.3 \%)$. Compared with arterial lactate, venous lactate predicted the composite neonatal outcome with comparable sensitivity $(75.0 \%$ versus $74.0 \%, \mathrm{P}>0.99)$, but slightly lower specificity (69.7\% versus $72.2 \%, \mathrm{P}<0.01)$.

Conclusion-Umbilical venous lactate strongly predicts arterial lactic acidemia and is comparable to arterial lactate for predicting neonatal morbidity at term. It could be used as a measure of neonatal morbidity when arterial blood is not available.

\section{INTRODUCTION}

Concentration of lactate, a direct end-product of anaerobic metabolism, in the umbilical artery has been used as a marker of fetal metabolic acidosis. Several studies have shown umbilical arterial lactate to be more sensitive and specific than umbilical artery $\mathrm{pH}$ and comparable to base excess for predicting short term neonatal morbidity [1-7]. Lactate has

\footnotetext{
Corresponding Author: Methodius G. Tuuli, MD,MPH, Washington University School of Medicine, Campus Box 8064, 4566 Scott Avenue, St. Louis, MO 63110, tuulim@ @udosis.wustl.edu.

Presented as at the $36^{\text {th }}$ Annual Meeting of the Society for Maternal-Fetal Medicine (SMFM), Atlanta, GA, and February 6, 2016.
} 
the additional advantage of being measured directly as opposed to base excess which is calculated using algorithms that may differ by the gas analyser used [8]. However it is not always feasible to obtain umbilical arterial blood at delivery [9-12]. Umbilical venous blood is much more readily obtainable because of the larger size of the umbilical vein. A recent study demonstrated venous blood gas parameters as powerful predictors of arterial blood gas $\mathrm{pH}$ and base excess, but did not assess lactate [13].

We conducted this prospective cohort study to estimate the utility of umbilical venous lactate for predicting arterial lactic acidemia and neonatal morbidity at term. We hypothesized that umbilical cord venous lactate is predictive of umbilical arterial lactic acidemia and neonatal morbidity at term.

\section{MATERIALS AND METHODS}

This was a prospective cohort study of consecutive deliveries at Washington University in St. Louis Medical Center from 2009 to 2014. The study was approved by the Washington University School of Medicine Human Research Protection Office.

Inclusion criteria were singleton pregnancies at term, vertex presentation, and labor prior to delivery. Multiple gestations and pregnancies with fetal anomalies were excluded. Term pregnancy was defined as gestational age $\geq 37$ weeks. Pregnancies were dated by a woman's last menstrual period and confirmed with first or second trimester ultrasound [14].

Demographic information, medical and surgical history, obstetric and gynecologic history, prenatal history and detailed labor and delivery information were abstracted from patients' charts by trained research nurses.

Umbilical cord blood was collected immediately after infant delivery, prior to knowledge of neonatal outcomes as previously described [7]. Briefly, a policy of universal umbilical cord gas and lactate measurement was instituted prior to the study. Both arterial and venous blood samples are obtained from a clamped segment of cord immediately after delivery. Umbilical blood lactate is measured from whole blood using an automated benchtop analyzer (DXC-800 Automated Chemistry Analyser, Beckman Coulter). As previously reported the coefficient of variation of the lactate assay in our laboratory is 2.9\% [7]. Umbilical arterial blood samples were validated to be arterial or venous by ensuring that $\mathrm{pH}$ was at least 0.02 lower in the artery than the vein [15].

The outcome measures were arterial lactic acidemia and a composite neonatal outcome. Umbilical arterial lactic acidemia was defined as arterial lactate $>3.9 \mathrm{mmol} / \mathrm{L}$ based on a prior study in our institution [7]. The composite neonatal outcome was made up of neonatal death and any of a number of neonatal morbidities including endotracheal intubation, mechanical ventilation, meconium aspiration syndrome, hypoxic-ischemic encephalopathy, and therapeutic hypothermia as previously reported [7]. Components of the composite were diagnosed by the attending neonatologist, without knowledge of the umbilical cord lactate levels. Meconium aspiration syndrome was diagnosed based on the presence of meconium stained amniotic fluid, neonatal respiratory distress, and characteristic radiographic abnormalities [16]. Hypoxic-ischemic encephalopathy was diagnosed based on the National 
Institute of Child Health and Human Development (NICHD) criteria [17]. Administration of therapeutic hypothermia was indicated for neonates meeting the following criteria per institutional protocol: $\geq 36$ weeks gestational age at birth, moderate to severe hypoxicischemic encephalopathy with or without seizures, and any one of 10-minute Apgar score $<5$, prolonged resuscitation at birth, severe acidosis $(\mathrm{pH}<7.1)$ on cord or neonate blood gas analysis within 60 minutes of birth, or base deficit $(>12 \mathrm{mmol} / \mathrm{L})$ on cord or neonate blood gas analysis within 60 minutes of birth [18]. Only one morbidity was counted per patient for the composite.

Baseline characteristics were calculated for the entire cohort and compared between women with and without the composite neonatal outcome. Continuous variables were compared using the Student's t test while categorical variables were compared using the chi-square or Fisher's exact test as appropriate. Normality of distribution of the continuous variables was evaluated using the Kolmogorov-Smirnov test.

We used linear regression analysis to examine the relationship between umbilical cord arterial and venous lactate. We constructed receiver-operating characteristics (ROC) curves to assess the predictive ability of umbilical venous lactate for arterial lactic acidemia, and to compare the predictive ability of venous and arterial lactate for the composite neonatal outcome. The 'optimal' cut-point of venous lactate for predicting arterial lactic acidemia was estimated based on the maximal Youden index [19]. The cut-point corresponding to the maximal Youden index maximizes the correct classification of subjects [20]. The area under the ROC curves for venous and arterial lactate were compared using the method described by Delong et al. [21]. We calculated and compared predictive characteristics (sensitivity, specificity, positive and negative predictive values, and positive and negative likelihod ratios) of arterial and venous lactate for neonatal morbidity based on the 'optimal' cut-

points. Sensitivities and specificities were compared using an extension of the McNemar test [22]. To explore the effects of using different cut-points of venous lactate on prediction of the composite neonatal outcome, we calculate predictive characteristics for different venous lactate cut-points. These included the $95^{\text {th }}$ and $99^{\text {th }}$ percentile thresholds from the current cohort as well as venous lactate thresholds estimated from arterial lactate thresholds in the literature $[2,23]$.

We did not estimate the sample size a priori; all consecutive patients meeting the inclusion criteria during the study period were included. All statistical tests were 2-tailed and $\mathrm{P}<0.05$ was considered significant. Analyses were conducted using STATA software package, version 12, Special Edition (College Station, TX).

\section{RESULTS}

A total of 8,580 singleton, term, non-anomalous births occurred during the study period. Patients who had missing or non-validated paired umbilical cord blood samples ( $\mathrm{n}=839$, 9.8\%) were excluded. The remaining 7,741 patients were included in the analysis (Fig. 1). The composite neonatal outcome was diagnosed in 104 neonates, constituting $1.3 \%$ of the cohort (Table 1). The most common morbidities were endotracheal intubation $(n=75)$ and 
mechanical ventilation $(n=50)$, followed by therapeutic hypothermia $(n=42)$ hypoxicischemic encephalopathy $(n=35)$ and meconium aspiration syndrome $(n=22)$.

Baseline characteristics were examined for the entire cohort and compared between those with and without the composite neonatal outcome (Table 2). The mean maternal age for the entire cohort was 25.7 years. The majority of the patients were African-American (68.9\%). The mean gestational age at delivery was 39.2 weeks. Women with and without the composite neonatal outcome were similar with regards to maternal age, racial composition, gestational age at delivery and birth weight. However, patients with the composite neonatal outcome had a higher mean body mass index ( 34.5 versus $32.3 \mathrm{Kg} / \mathrm{m} 2, \mathrm{p}<0.01)$, were significantly more likely to be nulliparous ( $46.2 \%$ versus $32.6 \%, \mathrm{p}<0.01)$, and deliver by cesarean $(50.0 \%$ versus $17.3 \%, \mathrm{p}<0.01)$. Patients with the composite neonatal outcome were also more likely to have chronic hypertension $(9.6 \%$ versus $4.8 \%, \mathrm{p}=0.02)$ and diabetes ( $9.6 \%$ versus $4.6 \%, \mathrm{p}=0.02$ ) Most baseline characteristics of the patients excluded were comparable to those of patients included in the study. In addition, incidence of the primary outcome in the patients excluded $(0.6 \%)$ was not significantly different from that in the patients included $(1.3 \%, \mathrm{P}=0.07)$.

There was a strong correlation between umbilical cord venous and arterial lactate (correlation coefficient $=0.916$ ). Arterial lactate increased by $1.04 \mathrm{mmol} / \mathrm{L}$ for every 1.00 $\mathrm{mmol} / \mathrm{L}$ increase in venous lactate (linear regression equation: arterial lactate $=0.22+1.04 \times$ venous lactate) (Fig. 2). The coefficient of determination $\left(R^{2}\right)$ was 0.839 , indicating that $83.9 \%$ of the variance in arterial lactate was explained by venous lactate. The mean difference between arterial and venous lactate was $0.34 \mathrm{mmol} / \mathrm{L}$ (95\% CI 0.32, 0.35).

Arterial lactic acidemia was identified in 2,199 neonates (28.4\%) based the arterial lactate cut-point of $3.9 \mathrm{mmol} / \mathrm{L}$. Venous lactate was highly predictive of arterial lactic acidemia (area under ROC curve: $0.958,95 \%$ CI $0.953,0.962$ ). The 'optimal' cut-point of venous lactate for predicting arterial lactic acidemia was $3.4 \mathrm{mmol} / \mathrm{L}$ based on the maximal Youden index (Fig. 3). This cut-point of venous lactate predicted arterial lactatic acidemia with a sensitivity of $87.0 \%$ (95\% CI 85.6\%, 88.4\%) and specificity of 91.3\% (95\% CI 90.6\%, $92.1 \%$ ). The positive and negative predictive values were $79.9 \%$ (95\% CI 78.3\%, 81.5\%) and $94.7 \%$ (95\% CI 94.0\%, 95.3\%), respectively.

Umbilical venous and arterial lactate were comparable for prediction of the composite neonatal morbidity (areas under ROC curve, 0.799 vs 0.815 , P=0.18) (Fig. 4). The 'optimal' cut-point of venous lactate for predicting the composite neonatal outcome was $3.4 \mathrm{mmol} / \mathrm{L}$, the same as that for predicting arterial lactic acidemia. The 'optimal' cut-point of arterial lactate for predicting the composite neonatal outcome was $3.8 \mathrm{mmol} / \mathrm{L}$ in this cohort, which is comparable to the previously published cut-point of $3.9 \mathrm{mmol} / \mathrm{L}$. Venous lactate predicted the composite neonatal outcome with comparable sensitivity as arterial lactate $(75.0 \%$ versus $74.0 \%, \mathrm{P}>0.99)$, but specificity was slightly lower $(69.7 \%$ versus $72.2 \%, \mathrm{P}<0.01)$. The associated negative and positive predictive values were nominally comparable (Table 3). Increasing cut-points of venous lactate had a predictable impact on the predictive characteristics. The most notable effects were a decrease in sensitivity, and an increase in specificity and negative predictive value (Table 4). 


\section{DISCUSSION}

We found that umbilical venous lactate was strongly predictive of arterial lactic acidemia. In addition, venous lactate predicted neonatal morbidity with comparable sensitivity as arterial lactate, but with slightly lower specificity.

The high predictive ability of venous lactate for arterial lactic acidemia and comparative prediction of neonatal morbidity is biologically plausible. While umbilical arterial lactate is a direct measure of fetal lactate and cord venous lactate may reflect placental lactate, the fetus has been identifed as the main source of umbilical cord lactate concentration in labor with little influence by maternal and uteroplacental production [24]. Thus, cord venous lactate after leaving the placenta remains predictive of arterial lactate.

Prior studies on lactate for predicting neonatal outcomes mostly compared umbilical arterial lactate to $\mathrm{pH}$ or base excess and many found higher sensitivity and specificity of arterial lactate over $\mathrm{pH}$, but comparable predictive characteristics as base excess [1-7]. These studies reported varying mean umbilical arterial lactate levels and proposed different cutpoints ranging from 3.2 to $10.0 \mathrm{mmol} / \mathrm{L}$ for predicting adverse outcomes [1, 25-27]. The differences are likely attributable to studies using hemolyzed or whole blood samples, and differences in lactate assays, study populations and outcome measures. While many collected paired arterial and venous lactate, they did not report the ability of venous lactate to predict arterial lactic acidemia or neonatal outcomes [12, 28, 29]. The differences between mean arterial and venous lactate levels were 0.2 to $0.5 \mathrm{mmol} / \mathrm{L}$, which is consistent with the $0.34 \mathrm{mmol} / \mathrm{L}$ we observed in the present study [12, 28, 29].

The large sample size with prospectively collected data is a major strength of this study. The universal institutional cord gas and lactate policy is important because it eliminates the selection bias inherent in selectively measuring cord gases in neonates at high risk for morbidity. We objectively validated samples as arterial and venous by ensuring that $\mathrm{pH}$ was at least 0.02 lower in the artery than the vein [15]. We used an objective statistical method to estimate and compare the predictive characteristics of the 'optimal' cut-points of venous and arterial lactate, but also explored the effects of using different cut-points on the predictive characteristics. In addition, we generated a regression equation that may be used to estimate arterial lactate for any given venous lactate.

We limited the study to term births following labor to control for variation of umbilical lactate with gestational age and labor [5, 28]. This makes our results not necessarily applicable to preterm births and deliveries occuring without labor. We used a composite neonatal outcome, which may be seen as a weakness. However, we chose components of the composite carefully, including only neonatal morbidities that are directly or indirectly linked to intrauterine hypoxia. Some baseline characteristics differed between patients with and without the composite neonatal outcome, reflecting that these factors predispose to neonatal morbidity. However, because we compared arterial and venous lactate in paired samples, it is likely that most of these factors would have similar effects on both values. Nearly $10 \%$ of patients were excluded for missing or non-validated paired lactate values. However, most baseline characteristics of the patients excluded were comparable to those included, 
suggesting a low risk of selection bias. Finally, we assessed only short term outcomes in this study.

In conclusion, results of this large prospective cohort study show that umbilical cord venous lactate is a strong predictor of umbilical arterial lactate with comparable predictive ability for neonatal morbidity as arterial lactate. The positive predictive value for the composite neonatal outcome was low, reflecting the low incidence of the outcome and suboptimal predictive ability of lactate for neonatal morbidity. Our results show that if lactate is used as a marker of neonatal morbidity, the predictive ability of venous and arterial lactate are comparable. As with all predictive studies, our findings should be validated in independent studies. These studies should also assess calibration of cut-points and neonatal outcome measures for desired sensitivity and specificity. Once validated, umbilical venous lactate could be a useful alternative measure of neonatal morbidity risk when arterial blood is not available. Importantly, our results show that the clinical value of measured umbilical cord lactate should not be discounted simply because its source is venous.

\section{Acknowledgments}

Dr. Tuuli was supported by a Women's Reproductive Health Research Career Development grant from the Eunice Kennedy Shriver National Institute of Child Health and Human Development (1K12HD063086-01). Dr. Cahill is supported by the Eunice Kennedy Shriver National Institute of Child Health and Human Development (R01HD061619-01, PI Cahill) and was a Robert Wood Johnson Foundation Faculty Physician Scholar, which partially supported this work. The contents of this publication are solely the responsibility of the authors and do not necessarily represent the official view of the NIH or Robert Wood Johnson Foundation.

\section{References}

1. Westgren M, et al. Routine measurements of umbilical artery lactate levels in the prediction of perinatal outcome. Am J Obstet Gynecol. 1995; 173(5):1416-22. [PubMed: 7503179]

2. Wiberg N, et al. Relation between umbilical cord blood $\mathrm{pH}$, base deficit, lactate, 5-minute Apgar score and development of hypoxic ischemic encephalopathy. Acta Obstet Gynecol Scand. 2010; 89(10):1263-9. [PubMed: 20846059]

3. Low JA, et al. Motor and cognitive deficits after intrapartum asphyxia in the mature fetus. Am J Obstet Gynecol. 1988; 158(2):356-61. [PubMed: 2449078]

4. Low JA, Panagiotopoulos C, Derrick EJ. Newborn complications after intrapartum asphyxia with metabolic acidosis in the term fetus. Am J Obstet Gynecol. 1994; 170(4):1081-7. [PubMed: 8166190]

5. Borruto F, et al. Screening of foetal distress by assessment of umbilical cord lactate. Clin Exp Obstet Gynecol. 2006; 33(4):219-22. [PubMed: 17211969]

6. Goldaber KG, et al. Pathologic fetal acidemia. Obstet Gynecol. 1991; 78(6):1103-7. [PubMed: 1945216]

7. Tuuli MG, et al. Umbilical cord arterial lactate compared with $\mathrm{pH}$ for predicting neonatal morbidity at term. Obstet Gynecol. 2014; 124(4):756-61. [PubMed: 25198278]

8. Wiberg N, Kallen K, Olofsson P. Base deficit estimation in umbilical cord blood is influenced by gestational age, choice of fetal fluid compartment, and algorithm for calculation. Am J Obstet Gynecol. 2006; 195(6):1651-6. [PubMed: 16996464]

9. Huisjes HJ, Aarnoudse JG. Arterial or venous umbilical pH as a measure of neonatal morbidity? Early Hum Dev. 1979; 3(2):155-61. [PubMed: 43802]

10. Armstrong L, Stenson BJ. Use of umbilical cord blood gas analysis in the assessment of the newborn. Arch Dis Child Fetal Neonatal Ed. 2007; 92(6):F430-4. [PubMed: 17951550]

11. Thorp JA, et al. Umbilical cord blood gas analysis at delivery. Am J Obstet Gynecol. 1996; 175(3 Pt 1):517-22. [PubMed: 8828408] 
12. White CR, et al. Benefits of introducing universal umbilical cord blood gas and lactate analysis into an obstetric unit. Aust N Z J Obstet Gynaecol. 2010; 50(4):318-28. [PubMed: 20716258]

13. Cantu J, et al. Predicting fetal acidemia using umbilical venous cord gas parameters. Obstet Gynecol. 2014; 124(5):926-32. [PubMed: 25437720]

14. Iams JD, et al. The Preterm Prediction Study: recurrence risk of spontaneous preterm birth. National Institute of Child Health and Human Development Maternal-Fetal Medicine Units Network. American journal of obstetrics and gynecology. 1998; 178(5):1035-40. [PubMed: 9609580]

15. Westgate J, Garibaldi JM, Greene KR. Umbilical cord blood gas analysis at delivery: a time for quality data. Br J Obstet Gynaecol. 1994; 101(12):1054-63. [PubMed: 7826958]

16. Fanaroff AA. Meconium aspiration syndrome: historical aspects. J Perinatol. 2008; 28(Suppl 3):S3-7. [PubMed: 19057607]

17. Shankaran $\mathrm{S}$, et al. Whole-body hypothermia for neonates with hypoxic-ischemic encephalopathy. N Engl J Med. 2005; 353(15):1574-84. [PubMed: 16221780]

18. Srinivasakumar $\mathrm{P}$, et al. Therapeutic hypothermia in neonatal hypoxic ischemic encephalopathy: electrographic seizures and magnetic resonance imaging evidence of injury. J Pediatr. 2013; 163(2):465-70. [PubMed: 23452588]

19. Youden WJ. Index for rating diagnostic tests. Cancer. 1950; 3(1):32-5. [PubMed: 15405679]

20. Zweig MH, Campbell G. Receiver-operating characteristic (ROC) plots: a fundamental evaluation tool in clinical medicine. Clinical chemistry. 1993; 39(4):561-77. [PubMed: 8472349]

21. DeLong ER, DeLong DM, Clarke-Pearson DL. Comparing the areas under two or more correlated receiver operating characteristic curves: a nonparametric approach. Biometrics. 1988; 44(3):83745. [PubMed: 3203132]

22. Lachenbruch PA, Lynch CJ. Assessing screening tests: extensions of McNemar's test. Stat Med. 1998; 17(19):2207-17. [PubMed: 9802179]

23. Kruger K, et al. Predictive value of fetal scalp blood lactate concentration and $\mathrm{pH}$ as markers of neurologic disability. Am J Obstet Gynecol. 1999; 181(5 Pt 1):1072-8. [PubMed: 10561620]

24. Nordstrom L, et al. Fetal and maternal lactate increase during active second stage of labour. BJOG. 2001; 108(3):263-8. [PubMed: 11281466]

25. Linet T, et al. [Microvolume dosage of lactate in cord blood for the evaluation of the neonatal wellbeing]. J Gynecol Obstet Biol Reprod (Paris). 2002; 31(4):352-7. [PubMed: 12058139]

26. Shirey T, St Pierre J, Winkelman J. Cord lactate, $\mathrm{pH}$, and blood gases from healthy neonates. Gynecol Obstet Invest. 1996; 41(1):15-9. [PubMed: 8821878]

27. Suidan JS, Young BK. Outcome of fetuses with lactic acidemia. Am J Obstet Gynecol. 1984; 150(1):33-7. [PubMed: 6433712]

28. Wiberg N, et al. Lactate concentration in umbilical cord blood is gestational age-dependent: a population-based study of 17867 newborns. BJOG. 2008; 115(6):704-9. [PubMed: 18410653]

29. Wiberg N, Kallen K, Olofsson P. Delayed umbilical cord clamping at birth has effects on arterial and venous blood gases and lactate concentrations. BJOG. 2008; 115(6):69-703. 


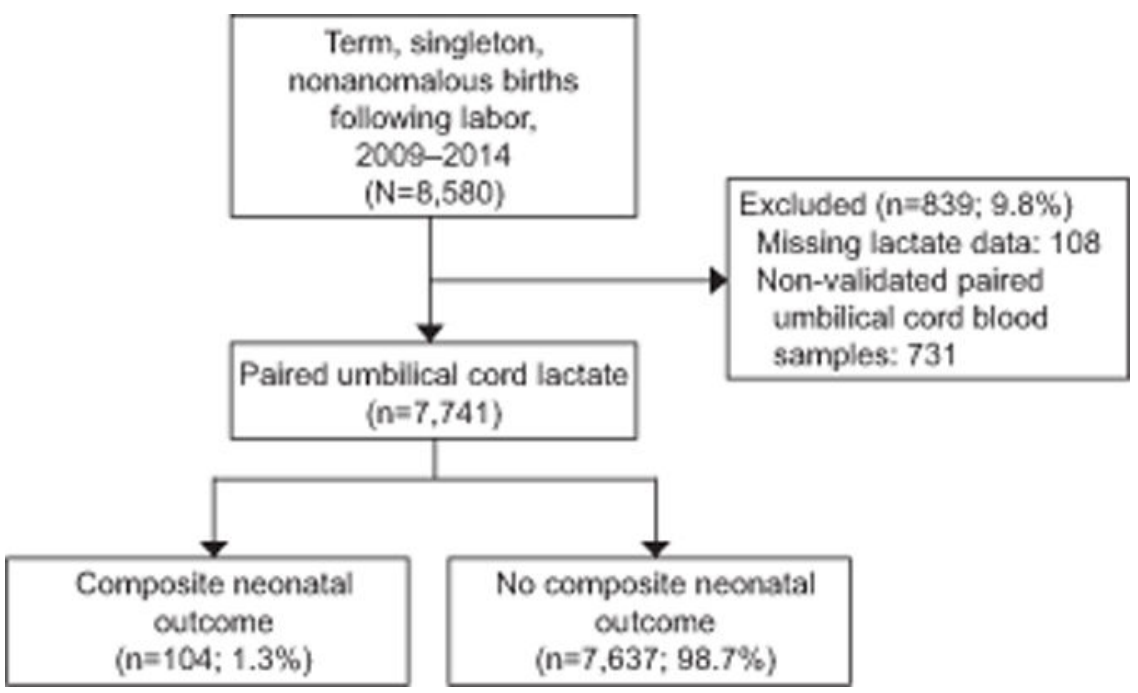

Figure 1.

Flow chart of study participants. 


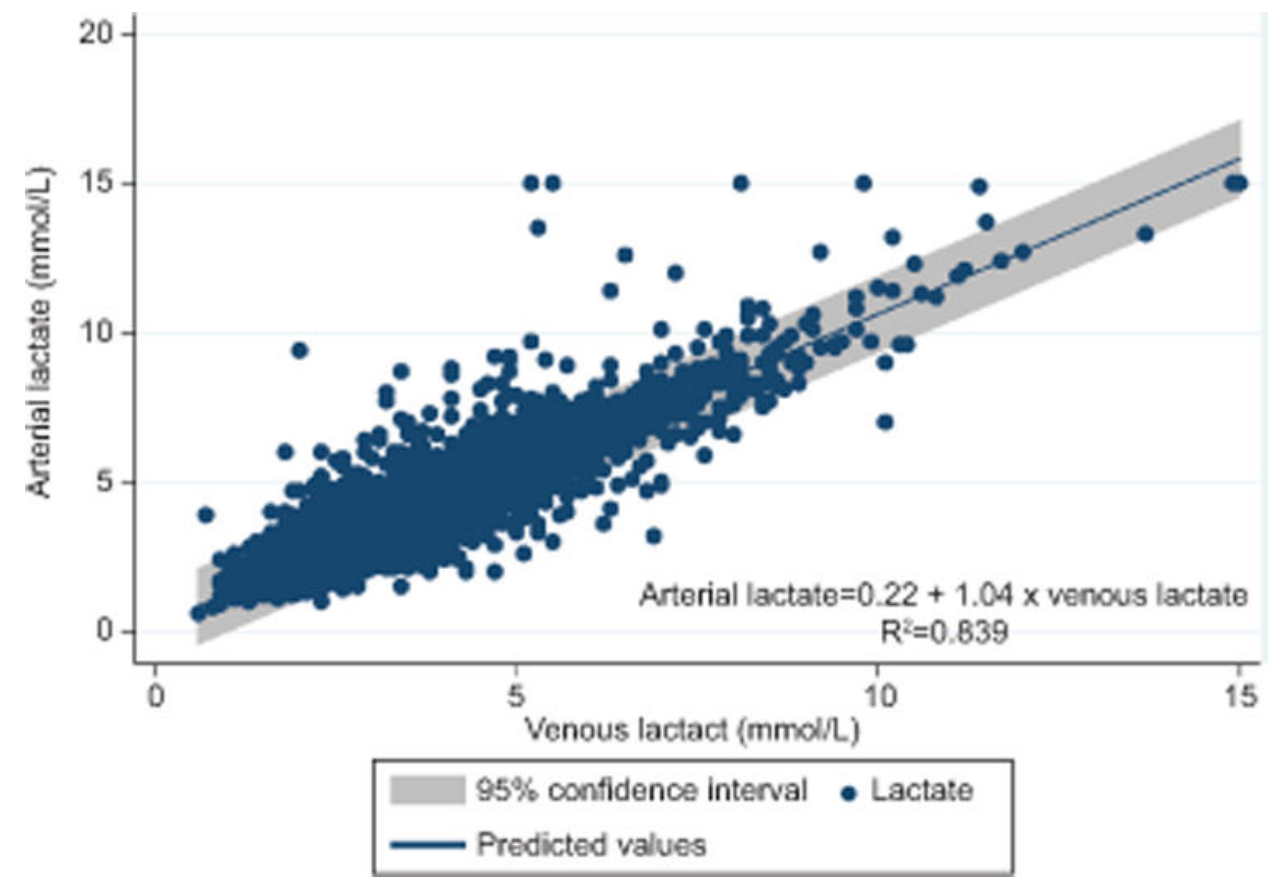

Figure 2.

Relationship between umbilical cord venous and arterial lactate levels $(n=7,741)$. 


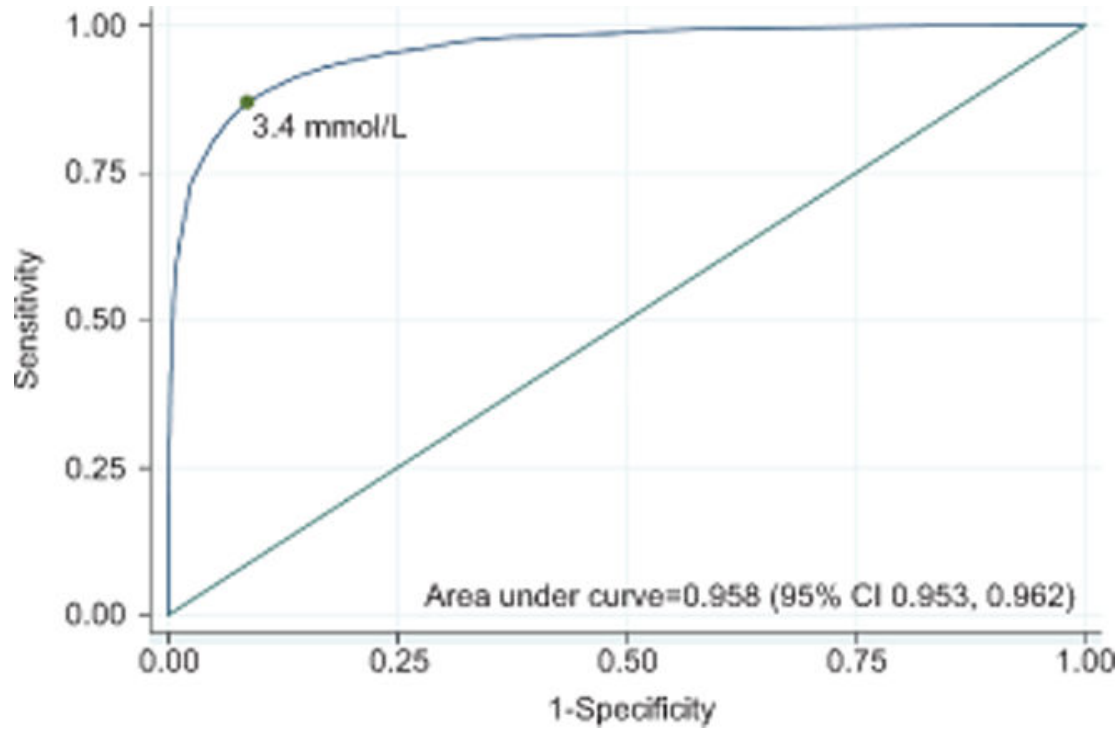

Figure 3.

Receiver-operating characteristics curve of umbilical venous lactate for predicting arterial lactic acidemia $(\mathrm{n}=7,741)$. The green dot, corresponding to a lactate level of $3.4 \mathrm{mmol} / \mathrm{L}$, represents the 'optimal' cut-point based on the maximal Youden index. CI, confidence interval. 


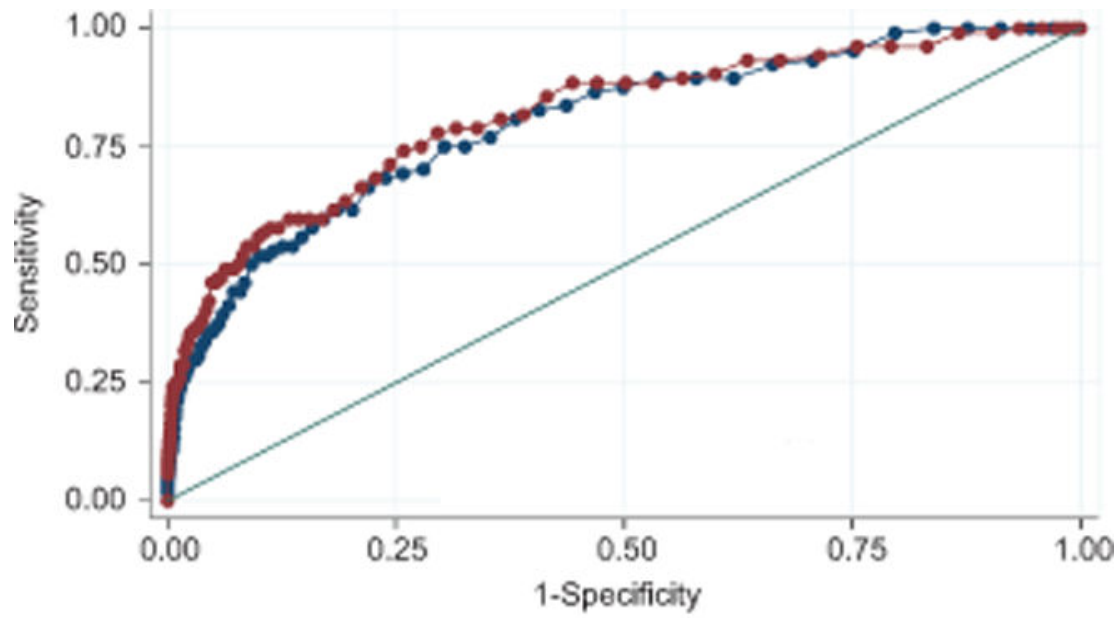
$P=18$

Figure 4.

Receiver-operating characteristics curve of umbilical cord venous and arterial lactate for predicting the composite neonatal outcome at term $(n=7,741)$. CI, confidence interval. 


\section{Table 1}

Distribution of components of the composite neonatal outcome ( $\mathrm{n}=104)$

\begin{tabular}{|l|c|}
\hline Component & *n (\%) \\
\hline Neonatal death & $4(3.8)$ \\
\hline Hypoxic-ischemic encephalopathy & $35(32.1)$ \\
\hline Therapeutic Hypothermia & $42(38.5)$ \\
\hline Endotracheal intubation & $75(68.8)$ \\
\hline Mechanical ventilation & $50(45.9)$ \\
\hline Meconium aspiration syndrome & $22(20.2)$ \\
\hline
\end{tabular}

* Some neonates were diagnosed with more than one outcome 
Table 2

Baseline characteristics of study participants $(n=7,741)$

\begin{tabular}{|c|c|c|c|c|}
\hline Variable & Entire Cohort $(\mathrm{N}=7,741)$ & $\begin{array}{c}\text { Neonatal Composite Morbidity } \\
(\mathrm{n}=104)\end{array}$ & $\begin{array}{l}\text { No Neonatal Composite } \\
\text { Morbidity }(n=7,637)\end{array}$ & ${ }^{*} \mathbf{P}$ \\
\hline Maternal age, years & $25.7 \pm 5.9$ & $25.9 \pm 6.7$ & $25.7 \pm 5.9$ & 0.78 \\
\hline \multicolumn{5}{|l|}{ Race } \\
\hline African American & $5053(68.9)$ & $65(64.4)$ & $4988(68.9)$ & \multirow[t]{3}{*}{0.59} \\
\hline Caucasian & $1732(23.6)$ & $28(27.7)$ & 1704 (23.6) & \\
\hline Hispanic & $551(7.5)$ & $8(7.9)$ & $543(7.5)$ & \\
\hline Body mass index, $\mathrm{Kg} / \mathrm{m} 2$ & $32.3 \pm 7.5$ & $34.5 \pm 8.2$ & $32.3 \pm 7.5$ & $<0.01$ \\
\hline Epidural & $6952(89.8)$ & $93(89.4)$ & $6859(89.8)$ & 0.90 \\
\hline Nulliparous & $2535(32.8)$ & $48(46.2)$ & 3487 (32.6) & $<0.01$ \\
\hline Smoking & $1074(13.9)$ & $16(15.4)$ & $1058(13.9)$ & 0.65 \\
\hline Alcohol & $86(1.1)$ & $3(2.9)$ & $83(1.1)$ & 0.11 \\
\hline Chronic hypertension & $369(4.8)$ & $10(9.6)$ & $359(4.8)$ & 0.02 \\
\hline Diabetes & $358(4.6)$ & $10(9.6)$ & $348(4.6)$ & 0.02 \\
\hline \multicolumn{5}{|l|}{ Mode of delivery } \\
\hline Spontaneous vaginal & $5996(77.5)$ & $46(44.2)$ & $5950(77.9)$ & \multirow[t]{3}{*}{$<0.01$} \\
\hline Operative vaginal & $372(4.8)$ & $6(5.8)$ & $366(4.8)$ & \\
\hline Cesarean & $1373(17.7)$ & $52(50.0)$ & $1321(17.3)$ & \\
\hline Gestational age, weeks & $39.2 \pm 1.2$ & $39.2 \pm 1.3$ & $39.2 \pm 1.2$ & 0.76 \\
\hline Birth weight, grams & $3250 \pm 464$ & $3275 \pm 516$ & $3250 \pm 463$ & 0.58 \\
\hline Small for gestational age & $1046(13.5)$ & $17(16.4)$ & 1029 (13.5) & 0.40 \\
\hline Male infant & $4022(52.0)$ & $65(62.5)$ & $3957(51.8)$ & 0.10 \\
\hline
\end{tabular}

BMI, body mass index; SD, standard deviation.

Data are mean \pm standard deviation or $\mathrm{n}(\%)$ unless otherwise specified.

* P-values based on the Student's t test for continuous variables and the chi-square or Fisher's exact test for categorical variables. 
Table 3

Predictive characteristics of 'optimal cut-points' of umbilical venous and arterial lactate predicting composite neonatal morbidity $(\mathrm{N}=7,741)$

\begin{tabular}{|l|c|c|c|}
\hline Characteristic & Venous lactate & Arterial Lactate & \multirow{2}{*}{ P } \\
\cline { 1 - 3 } Optimal cut-point & $\mathbf{3 . 4} \mathbf{~ m m o l} / \mathbf{L}$ & $\mathbf{3 . 9} \mathbf{~ m m o l} / \mathbf{L}$ & \\
\hline Sensitivity (95\% CI) & $75.0(65.3,83.1)$ & $74.0(64.3,82.3)$ & $>0.99$ \\
\hline Specificity (95\% CI) & $69.7(68.6,70.7)$ & $72.2(71.2,73.2)$ & $<0.01$ \\
\hline Positive predictive value (95\% CI) & $3.1(2.5,3.9)$ & $2.9(2.7,4.2)$ & - \\
\hline Negative predictive value $(95 \% \mathrm{CI})$ & $99.8(99.5,99.9)$ & $99.6(99.3,99.8)$ & - \\
\hline Positive likelihood ratio $(95 \% \mathrm{CI})$ & $2.5(2.2,2.8)$ & $2.7(2.4,3.0)$ & - \\
\hline Negative likelihood ratio $(95 \% \mathrm{CI})$ & $0.4(0.3,0.50)$ & $0.4(0.3,0.5)$ & - \\
\hline
\end{tabular}




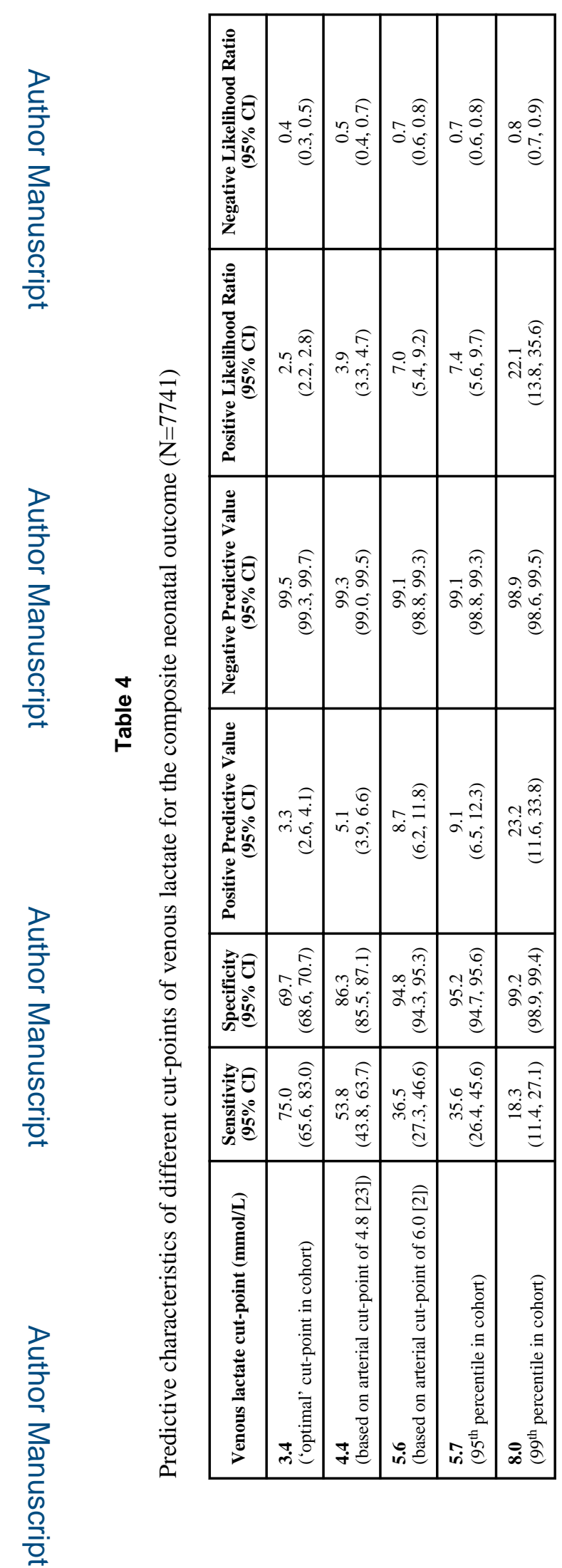

Obstet Gynecol. Author manuscript; available in PMC 2017 April 01. 